\title{
Liouville-type theorems for a quasilinear elliptic equation of the Hénon-type
}

\author{
Quoc Hung Phan and Anh Tuan Duong
}

\begin{abstract}
We consider the Hénon-type quasilinear elliptic equation $-\Delta_{m} u=|x|^{a} u^{p}$ where $\Delta_{m} u=\operatorname{div}\left(|\nabla u|^{m-2} \nabla u\right), m>1, p>m-1$ and $a \geq 0$. We are concerned with the Liouville property, i.e. the nonexistence of positive solutions in the whole space $\mathbb{R}^{N}$. We prove the optimal Liouville-type theorem for dimension $N<m+1$ and give partial results for higher dimensions.
\end{abstract}

Mathematics Subject Classification. Primary 35B53, 35J62; Secondary 35K57, 35B33.

Keywords. Quasilinear, Liouville-type theorem, Hénon-type equation.

\section{Introduction}

This article is devoted to the study of positive solutions of the following elliptic equation

$$
-\Delta_{m} u=|x|^{a} u^{p}, \quad x \in \Omega,
$$

where $\Delta_{m} u=\operatorname{div}\left(|\nabla u|^{m-2} \nabla u\right)$ denotes the $m$-Laplace operator, $\Omega$ is a domain of $\mathbb{R}^{N}$. We assume throughout the paper that

$$
1<m<p+1, \quad \text { and } \quad a \geq 0 .
$$

The interest of Eq. (1.1) started from the case of classical Laplacian

$$
-\Delta u=|x|^{a} u^{p},
$$

which is called the Hénon equation. Since the pioneering work of Hénon [12] in 1973 on the studying of rotating stellar structures, a variety of results on the qualitative properties of the solutions to problem (1.2) have been established. In particular, the results on the existence and nonexistence, the multiplicity, the symmetry-breaking properties, and blow-up profile of solutions were obtained - see $[2-4,17,23,26,27]$. 
The problem (1.1) for $m \neq 2$ arises in the theory of quasi-regular and quasi-conformal mappings, and in mathematical modelling of non-Newtonian fluids. Media with $m>2$ and $m<2$ are called dilatant fluids and pseudoplastics respectively (see the references in [25] for a discussion of the physical background).

In general case of $m$, the existence and nonexistence results were widely studied. Among others, Clement et al. [7] applied the mountain pass theorem and proved that the boundary value problem possesses at least a radial solution for all $m<p<p_{S}(m, a)$, extending Ni's result [17] for the more general class of equations. Carriao et al. [5] proved some existence and multiplicity results of non-radial solutions. The nonexistence of nontrivial solutions was established via the generalized Pohozaev identity, see $[10,11,18]$. Further results on he asymptotic behaviour of solutions near the singularity and qualitative properties of bounded radial ground states can be found in $[1,21,22]$.

The aim of this paper is to study the Liouville-type theorems for the problem (1.1). Before stating our main results, let us introduce the following exponents

$$
\begin{aligned}
p_{S}(m, a) & :=\frac{(m-1) N+m}{N-m+m a} \quad(=\infty \quad \text { if } N \leq m), \\
p_{S}(m) & :=p_{S}(m, 0) .
\end{aligned}
$$

Our notion of solution is that of continuously differentiable weak solution, which is defined as follows

Definition 1.1. For an arbitrary domain $\Omega$ of $\mathbb{R}^{N}$, we say that a nonnegative function $u$ is a solution of (1.1) if it satisfies

$$
u \in C^{1}(\Omega), \quad \int_{\Omega}|\nabla u|^{m-2} \nabla u . \nabla \varphi d x=\int_{\Omega}|x|^{a} u^{p} \varphi d x \quad \text { for all } \varphi \in C_{0}^{\infty}(\Omega) .
$$

Roughly speaking, a (continuously differentiable weak) solution of (1.1) is a $C^{1}$-function which solves (1.1) in the distributional sense.

We recall that Liouville-type theorem is the nonexistence of solution in the entire space. The classical Liouville-type theorem stated that a bounded harmonic (or holomorphic) function defined in entire space must be constant. This theorem, known as Liouville Theorem, was first announced in 1844 by Liouville [15] for the special case of a doubly-periodic function. Later in the same year, Cauchy [6] published the first proof of the above stated theorem. In 1981, Gidas and Spruck established in pioneering article [9] the optimal Liouville-type result for nonnegative solutions of the semilinear elliptic equation $-\Delta u=u^{p}$. Since then, the Liouville property has been refined considerably and emerged as one of the most powerful tools in the study of boundary value problems for nonlinear PDEs (see e.g. [20]).

Concerning the Liouville-type results for the problem (1.1), the case $a=0$ was completely established by Serrin and Zou [25]. Here, the optimal Liouvilletype theorem states that the Eq. (1.1) has no positive solution in $\Omega=\mathbb{R}^{N}$ if and only if $p<p_{S}(m)$. 
The case $a>0$ is less understood, it seems that the presence of the term $|x|^{a}$ modifies the range of values of $p$ for the non-existence of entire positive solutions. In the class of radial solutions, the Liouville property was completely solved (see e.g. [14, Section 3]). More precisely,

Proposition A. (i) If $p<p_{S}(m, a)$, then Eq. (1.1) has no positive radial solution in $\Omega=\mathbb{R}^{N}$.

(ii) If $p \geq p_{S}(m, a)$, then Eq. (1.1) possesses a bounded, positive radial solution in $\Omega=\mathbb{R}^{N}$.

The exponent $p_{S}(m, a)$ thus plays a critical role in the radial case and this, in addition to the above mentioned result for $a=0$, supports the following natural conjecture:

Conjecture B. If $p<p_{S}(m, a)$, then Eq. (1.1) has no positive solutions in $\Omega=\mathbb{R}^{N}$.

The condition $p<p_{S}(m, a)$ is optimal due to Proposition A(ii). However, apart from the radial case, the best available condition on $p$ for the nonexistence of entire positive solutions up to now is as follows (e.g. [16, Theorem 12.4])

$$
p \leq \frac{(m-1)(N+a)}{N-m} \quad(p<\infty \quad \text { if } N \leq m)
$$

In fact, Eq. (1.4) is the optimal condition for the nonexistence of supersolutions (i.e. solution to $-\Delta_{m} u \geq|x|^{a} u^{p}$ ) in $\mathbb{R}^{N}$, or in an exterior domain. This result in particular implies that the Conjecture $\mathrm{B}$ is true for the dimension $N \leq m$. The aim of this paper is to prove Conjecture B for dimension $N<m+1$. Our main result is the following.

Theorem 1.2. Let $N<m+1$. If $p<p_{S}(m, a)$, then Eq. (1.1) has no positive solution in $\Omega=\mathbb{R}^{N}$.

We also have the following partial result for higher dimensions.

Theorem 1.3. If $p<p_{S}(m)$ then Eq. (1.1) has no positive solution in $\Omega=\mathbb{R}^{N}$.

Remark 1.4. (a) We stress that there is no boundedness assumption on solutions in Theorems 1.2 and 1.3.

(b) The proof of Theorem 1.2 uses the technique introduced by Serrin and Zou [24] and further developed by Souplet [28], which is based on a combination of Pohozaev identity, Sobolev inequality on the unit sphere $S^{N-1}$ and measure and feedback arguments. However, we point out that some additional difficulties arise in our problem. For instance, the very technical measure and feedback arguments become even more complicated since the lack of regularity and interpolation inequalities for the $m$-Laplacian. The presence of weight functions makes the problem much more delicate. Moreover, one can not apply the embedding $W^{2,1+\varepsilon}\left(S^{N-1}\right) \subset$ $L^{\infty}\left(S^{N-1}\right)$ as in the case of Laplace operator. We instead use the embed$\operatorname{ding} W^{1, m}\left(S^{N-1}\right) \subset L^{\infty}\left(S^{N-1}\right)$ and combine this with some additional arguments. 
As applications of Liouville-type theorems, we provide some results on singularity and decay estimates:

Proposition 1.5. Let $m-1<p<p_{S}(m)$. There exists a positive constant $C=C(N, p, m, a)$ such that the following assertions hold.

(i) Any nonnegative solution of Eq. (1.1) in $\Omega=\left\{x \in \mathbb{R}^{N} ; 0<|x|<\rho\right\}$ $(\rho>0)$ satisfies

$$
u(x) \leq C|x|^{-\frac{m+a}{p+1-m}} \quad \text { and } \quad|\nabla u(x)| \leq C|x|^{-\frac{p+1+a}{p+1-m}}, \quad 0<|x|<\rho / 2 .
$$

(ii) Any nonnegative solution of $E q$. (1.1) in $\Omega=\left\{x \in \mathbb{R}^{N} ;|x|>\rho\right\}(\rho \geq 0)$ satisfies

$$
u(x) \leq C|x|^{-\frac{m+a}{p+1-m}} \quad \text { and } \quad|\nabla u(x)| \leq C|x|^{-\frac{p+1+a}{p+1-m}}, \quad|x|>2 \rho .
$$

Our proof of Proposition 1.5 is based on the observation that estimates (1.5) and (1.6) for given $p, a$ can be rather easily reduced to the Liouville property for the same $p$ but with a replaced by 0 . This reduction relies on two ingredients:

(i) a change of variable, that allows to replace the coefficient $|x|^{a}$ with a smooth function which is bounded and bounded away from 0 in a suitable spatial domain;

(ii) a generalization of a doubling-rescaling argument from [20].

We can then obtain an easy derivation of Theorem 1.3 from Proposition 1.5, by combining the Pohozaev identity with the decay estimate (1.6). We note that the gradient part of estimate (1.6) is crucial for the proof in order to estimate some of the terms appearing in the Pohozaev identity.

The rest of the paper is organized as follows. In Sect. 2, we recall some basic estimates and identities. Section 3 is devoted to the delicate proof of Theorem 1.2. Finally, in "Appendix", we collect the proofs of some results which we use and are more or less known, but whose proofs we prefer to provide for completeness. This includes Proposition 1.5 and Theorem 1.3.

\section{Preliminaries}

For $R>0$, we set $B_{R}=\left\{x \in \mathbb{R}^{N}:|x|<R\right\}$. We shall use spherical coordinates $r=|x|, \theta=x /|x| \in S^{N-1}$ and write $u=u(r, \theta)$. The surface measures on $S^{N-1}$ and on the sphere $\left\{x \in \mathbb{R}^{N}:|x|=R\right\}, R>0$, will be denoted respectively by $d \theta$ and by $d \sigma_{R}$. For given function $w=w(\theta)$ on $S^{N-1}$ and $1 \leq k \leq \infty$, we set $\|w\|_{k}=\|w\|_{L^{k}\left(S^{N-1}\right)}$. When no confusion is likely, we shall denote $\|u\|_{k}=\|u(r, \cdot)\|_{k}$ and $\nabla_{x} u=\nabla u$.

We first recall the following fundamental Sobolev inequality (see e.g. [24]).

Lemma 2.1. (Sobolev inequalities on $S^{N-1}$ ) Let $N \geq 2, j \geq 1$ is integer and $1<k<\lambda \leq \infty, k \neq(N-1) / j$. For $w=w(\theta) \in W^{j, k}\left(S^{N-1}\right)$, we have

$$
\|w\|_{\lambda} \leq C\left(\left\|D_{\theta}^{j} w\right\|_{k}+\|w\|_{1}\right)
$$


where

$$
\begin{cases}\frac{1}{k}-\frac{1}{\lambda}=\frac{j}{N-1} & \text { if } k<(N-1) / j, \\ \lambda=\infty & \text { if } k>(N-1) / j\end{cases}
$$

and $C=C(j, k, N)>0$.

Setting

$$
\alpha=\frac{m+a}{p+1-m}
$$

we have the following basic integral estimates for solutions to (1.1) in $\mathbb{R}^{N}$.

Lemma 2.2. Let $u$ be a positive solution of (1.1) in $\Omega=\mathbb{R}^{N}$. Then there holds

$$
\int_{B_{R} \backslash B_{R / 2}} u^{p} d x \leq C R^{N-p \alpha}, \quad R>0,
$$

with $C=C(N, p, m, a)>0$.

The proof of Lemma 2.2 is totally similar to that of [16, Theorem 12.1] where the authors proved for the case $a=0$ by using rescaled test function argument. From Lemma 2.2, by interpolation, one can deduce the following corollary.

Corollary 2.3. Let $u$ be a positive solution of (1.1) in $\Omega=\mathbb{R}^{N}$. For $R>0$ and $0 \leq q \leq p$, we have

$$
\int_{B_{R} \backslash B_{R / 2}} u^{q} d x \leq C R^{N-q \alpha},
$$

with $C=C(N, p, q, m, a)>0$.

Next, we need the following estimate for the proof of Theorem 1.2.

Lemma 2.4. Let $\varepsilon>0$ such that $m>1+\varepsilon$. If $u$ is positive solution of (1.1) in $\Omega=\mathbb{R}^{N}$, then there exists $C=C(N, p, m, a, \varepsilon)>0$ such that

$$
\int_{B_{R} \backslash B_{R / 2}} \frac{|\nabla u|^{m}}{u^{1+\varepsilon}} d x \leq C R^{N-m-(m-1-\varepsilon) \alpha} .
$$

Proof. Fix $\phi \in \mathcal{D}\left(\mathbb{R}^{N}\right), 0 \leq \phi \leq 1$ such that $\phi(x)=1$ for $|x| \leq 1$ and $\phi(x)=0$ for $|x|>2,|\nabla \phi| \leq C \phi$. For each $R>0$, put $\phi_{R}(x)=\phi(x / R)$. We have

$$
\left|\nabla \phi_{R}(x)\right| \leq C R^{-1} \phi_{R}
$$

Since $u$ is a distributional solution, we thus have

$$
\begin{aligned}
\int_{\mathbb{R}^{N}}|x|^{a} u^{p-\varepsilon} \phi_{R} d x & =\int_{\mathbb{R}^{N}}|\nabla u|^{m-2} \nabla u \cdot \nabla\left(u^{-\varepsilon} \phi_{R}\right) d x \\
& =-\varepsilon \int_{\mathbb{R}^{N}} \frac{|\nabla u|^{m}}{u^{1+\varepsilon}} \phi_{R} d x+\int_{\mathbb{R}^{N}} u^{-\varepsilon}|\nabla u|^{m-2} \nabla u . \nabla \phi_{R} d x .
\end{aligned}
$$


Hence,

$$
\begin{aligned}
\int_{\mathbb{R}^{N}} \frac{|\nabla u|^{m}}{u^{1+\varepsilon}} \phi_{R} d x & =-\frac{1}{\varepsilon} \int_{\mathbb{R}^{N}}|x|^{a} u^{p-\varepsilon} \phi_{R} d x+\frac{1}{\varepsilon} \int_{\mathbb{R}^{N}} u^{-\varepsilon}|\nabla u|^{m-2} \nabla u . \nabla \phi_{R} d x \\
& \leq \frac{C}{R} \int_{R<|x|<2 R} u^{-\varepsilon}|\nabla u|^{m-1} \phi_{R} d x .
\end{aligned}
$$

Applying Young's inequality

$$
\begin{aligned}
\frac{C}{R} u^{-\varepsilon}|\nabla u|^{m-1} & =\frac{|\nabla u|^{m-1}}{u^{\frac{(1+\varepsilon)(m-1)}{m}}} \cdot \frac{C}{R} u^{\frac{m-1-\varepsilon}{m}} \\
& \leq \frac{m-1}{m} \frac{|\nabla u|^{m}}{u^{1+\varepsilon}}+\frac{1}{m} \frac{C^{m}}{R^{m}} u^{m-1-\varepsilon}
\end{aligned}
$$

Combining (2.5) with (2.6), we have

$$
\begin{aligned}
\int_{\mathbb{R}^{N}} \frac{|\nabla u|^{m}}{u^{1+\varepsilon}} \phi_{R} d x & \leq \frac{C}{R^{m}} \int_{R<|x|<2 R} u^{m-1-\varepsilon} \phi_{R} d x \\
& \leq \frac{C}{R^{m}} \int_{R<|x|<2 R} u^{m-1-\varepsilon} d x \leq C R^{N-m-(m-1-\varepsilon) \alpha}
\end{aligned}
$$

Therefore

$$
\int_{B_{R}} \frac{|\nabla u|^{m}}{u^{1+\varepsilon}} d x \leq C R^{N-m-(m-1-\varepsilon) \alpha}
$$

The following Pohozaev identity plays a key role in the proof of Theorems 1.2 and 1.3. It is probably known, but we give a proof for completeness, especially since there is a slight technical difficulty arising from the regularity of $m$-Laplacian.

Lemma 2.5. (Pohozaev identity) Let $u$ be a positive solution of (1.1) in $\mathbb{R}^{N}$. For all $R>0$, there holds

$$
\begin{aligned}
& \left(m \frac{N+a}{p+1}-N+m\right) \int_{B_{R}}|x|^{a} u^{p+1} d x \\
& =\int_{|x|=R}\left(m R^{1+a} \frac{u^{p+1}}{p+1}+m R\left|u^{\prime}\right|^{2}|\nabla u|^{m-2}-R|\nabla u|^{m}\right) d \sigma_{R} \\
& \quad+\int_{|x|=R}(N-m) u u^{\prime}|\nabla u|^{m-2} d \sigma_{R},
\end{aligned}
$$

where $u^{\prime}=\frac{x}{|x|} \cdot \nabla u$ and $\nabla u=\nabla_{x} u$.

For the proof of the Pohozaev identity, we first recall the following standard property (e.g. [13, Lemma A.1]).

Lemma 2.6. [13] Let $\Omega$ be a bounded domain in $\mathbb{R}^{N}$ with a $C^{2}$-boundary $\partial \Omega$. Assume that $\mathbf{a}: \Omega \rightarrow \mathbb{R}^{N}$ satisfies $\mathbf{a} \in\left[C^{0}(\Omega)\right]^{N}$ and $\operatorname{div} \mathbf{a}=f \in L^{1}(\Omega)$ in the 
sense of distributions in $\Omega$.Then we have

$$
\int_{\partial \Omega} \mathbf{a}(x) \cdot \nu(x) d \sigma(x)=\int_{\Omega} f(x) d x .
$$

Proof of Lemma 2.5. It follows from [13, Theorem 4.2] that

$$
\begin{aligned}
& (N-m) \int_{B_{R}}|\nabla u|^{m} d x+m \int_{B_{R}}|x|^{a} u^{p}(x . \nabla u) d x \\
& \quad=R \int_{\partial B_{R}}|\nabla u|^{m} d \sigma_{R}-m R \int_{\partial B_{R}}\left|u^{\prime}\right|^{2}|\nabla u|^{m-2} d \sigma_{R} .
\end{aligned}
$$

Since

$$
(x . \nabla u)|x|^{a} u^{p}=-\frac{N+a}{p+1}|x|^{a} u^{p+1}+\operatorname{div}\left(x|x|^{a} \frac{u^{p+1}}{p+1}\right),
$$

we have,

$$
\int_{B_{R}}|x|^{a} u^{p}(x . \nabla u) d x=-\frac{N+a}{p+1} \int_{B_{R}}|x|^{a} u^{p+1} d x+R^{1+a} \int_{|x|=R} \frac{u^{p+1}}{p+1} d \sigma_{R} .
$$

Now, let $\mathbf{a}=u|\nabla u|^{m-2} \nabla u$ and note that $\operatorname{div} \mathbf{a}=-|x|^{a} u^{p+1}+|\nabla u|^{m}$ in sense of distribution. Lemma 2.6 implies

$$
\int_{\partial B_{R}} u u^{\prime}|\nabla u|^{m-2} d \sigma_{R}=\int_{B_{R}}\left(-|x|^{a} u^{p+1}+|\nabla u|^{m}\right) d x .
$$

Consequently,

$$
\int_{B_{R}}|\nabla u|^{m} d x=\int_{B_{R}}|x|^{a} u^{p+1} d x+\int_{\partial B_{R}} u u^{\prime}|\nabla u|^{m-2} d \sigma_{R} .
$$

Lemma 2.5 follows from (2.8) to (2.10).

\section{Proof of Theorem 1.1}

Proof of Theorem 1.2. Since Theorem 1.2 was proved for dimension $N \leq m$ (cf. [16]), we assume that $N>m$. Suppose there exists positive solution $u$ to (1.1) in $\mathbb{R}^{N}$. We fix a number $\varepsilon>0$ such that $m-1-\varepsilon>0$. In what follows, $C$ denotes any positive constant independent of $R$ (but possibly depending on $\varepsilon)$.

Step 1: preparations. Let $F(R)=\int_{B_{R}}|x|^{a} u^{p+1} d x$. By using Pohozaev identity we can deduce

$$
F(R) \leq C\left(G_{1}(R)+G_{2}(R)+G_{3}(R)\right)
$$


where

$$
\begin{aligned}
& G_{1}(R)=R^{N+a} \int_{S^{N-1}} u^{p+1}(R, \theta) d \theta, \\
& G_{2}(R)=R^{N} \int_{S^{N-1}}\left|\nabla_{x} u(R, \theta)\right|^{m} d \theta, \\
& G_{3}(R)=R^{N-m} \int_{S^{N-1}} u^{m}(R, \theta) d \theta .
\end{aligned}
$$

Step 2: estimation of $G_{1}(R)$ and $G_{2}(R)$ in terms of suitable norms. Let

$$
v=u^{\frac{m-1-\varepsilon}{m}} \text {. }
$$

Recall that $\|v\|_{k}$ denotes $\|v(R, \cdot)\|_{L^{k}\left(S^{N-1}\right)}$. Since $N-1<m$, then the embedding $W^{1, m}\left(S^{N-1}\right) \subset L^{\infty}\left(S^{N-1}\right)$ is continuous. By Lemma 2.1, we have

$$
\|v\|_{\infty} \leq C\left(\left\|D_{\theta} v\right\|_{m}+\|v\|_{1}\right) \leq C\left(R\left\|\nabla_{x} v\right\|_{m}+\|v\|_{1}\right) .
$$

Therefore,

$$
\begin{aligned}
G_{1}(R) & \leq C R^{N+a}\|u\|_{\infty}^{p+1} \\
& \leq C R^{N+a}\|v\|_{\infty}^{(p+1) m /(m-1-\varepsilon)} \\
& \leq C R^{N+a}\left(R\left\|\nabla_{x} v\right\|_{m}+\|v\|_{1}\right)^{(p+1) m /(m-1-\varepsilon)} .
\end{aligned}
$$

Similarly,

$$
G_{3}(R) \leq C R^{N-m}\left(R\left\|\nabla_{x} v\right\|_{m}+\|v\|_{1}\right)^{m^{2} /(m-1-\varepsilon)} .
$$

Next,

$$
\begin{aligned}
G_{2}(R) & =R^{N} \int_{S^{N-1}}\left|\nabla_{x} u(R, \theta)\right|^{m} d \theta \\
& =C R^{N} \int_{S^{N-1}}\left|\nabla_{x} v(R, \theta)\right|^{m} v^{\frac{m+m \varepsilon}{m-1-\varepsilon}}(R, \theta) d \theta \\
& \leq C R^{N}\left\|\nabla_{x} v\right\|_{m}^{m}\|v\|_{\infty}^{\frac{m+m \varepsilon}{m-1-\varepsilon}} .
\end{aligned}
$$

Step 3: control of the averages and measure argument. For any $R>1$, denote $\beta=\frac{m-1-\varepsilon}{m} \alpha$. It follows from Corollary 2.3 and Lemma 2.4 that

$$
\int_{R / 2}^{R}\|v(r)\|_{1} r^{N-1} d r \leq C R^{N-\beta}
$$

and

$$
\int_{R / 2}^{R}\left\|\nabla_{x} v(r)\right\|_{m}^{m} r^{N-1} d r \leq C R^{N-m-m \beta} .
$$

For a given $K>0$, let us define the sets

$$
\begin{aligned}
& \Gamma_{1}(R)=\left\{r \in(R, 2 R) ;\|v(r)\|_{1}>K R^{-\beta}\right\}, \\
& \Gamma_{2}(R)=\left\{r \in(R, 2 R) ;\left\|\nabla_{x} v(r)\right\|_{m}^{m}>K R^{-m-m \beta}\right\} .
\end{aligned}
$$


By estimate (3.7), for $R>1$, we have

$$
\begin{aligned}
C & \geq R^{-N+\beta} \int_{R}^{2 R}\|v(r)\|_{1} r^{N-1} d r \\
& \geq R^{-N+\beta}\left|\Gamma_{1}(R)\right| R^{N-1} K R^{-\beta}=K\left|\Gamma_{1}(R)\right| R^{-1} .
\end{aligned}
$$

Consequently, $\left|\Gamma_{1}\right| \leq R / 4$ for $K \geq 4 C$. Similarly, from estimates (3.8), we obtain $\left|\Gamma_{2}\right| \leq R / 4$. Hence, for each $R \geq 1$, we can assert the existence of

$$
\tilde{R} \in(R, 2 R) \backslash \bigcup_{i=1}^{2} \Gamma_{i}(R) \neq \emptyset .
$$

Step 4: conclusion. It follows from (3.4) to (3.6) in Step 2 and (3.9) in Step 3 that

$$
\begin{aligned}
& G_{1}(\tilde{R}) \leq C R^{N+a}\left(R^{-\beta}\right)^{(p+1) m /(m-1-\varepsilon)}=C R^{N+a-\alpha(p+1)}, \\
& G_{3}(\tilde{R}) \leq C R^{N-m-\frac{\beta m^{2}}{m-1-\varepsilon}}=C R^{N-m-m \alpha}
\end{aligned}
$$

and

$$
G_{2}(\tilde{R}) \leq C R^{N-m-m \beta-\frac{m+m \varepsilon}{m-1-\varepsilon} \beta}=C R^{N-m-m \alpha} .
$$

We note that $N+a-\alpha(p+1)=N-m-m \alpha:=\tilde{a}$. By straightforward computation, we see that $p<p_{S}(m, a)$ is equivalent to $\tilde{a}<0$. From (3.10) to (3.11), we have

$$
F(R) \leq F(\tilde{R}) \leq C R^{\tilde{a}}, \quad R \geq 1
$$

Therefore, let $R \rightarrow \infty$ we obtain $\int_{\mathbb{R}^{N}}|x|^{a} u^{p+1} d x=0$, hence $u \equiv 0$ : a contradiction. The proof is complete.

\section{Acknowledgments}

The first author would like to thank the Vietnam Institute for Advanced Study in Mathematics (VIASM) for the hospitality during the completion of this work. This research is funded by Vietnam National Foundation for Science and Technology Development (NAFOSTED) under Grant No. 101.02-2014.06.

\section{Appendix}

\section{Singularity and decay estimates}

We shall prove Proposition 1.5. First, we need the following lemma which is analogous to [19, Lemma 2.1].

Lemma 4.1. Let $1<p<p_{S}(m)$ and $\gamma \in(0,1]$. Let $c \in C^{\gamma}\left(\bar{B}_{1}\right)$ satisfy

$$
\|c\|_{C^{\gamma}\left(\bar{B}_{1}\right)} \leq C_{1} \quad \text { and } \quad c(x) \geq C_{2}, \quad x \in \bar{B}_{1},
$$

for some constants $C_{1}, C_{2}>0$. There exists a constant $C$, depending only on $\gamma, C_{1}, C_{2}, p, m, N$, such that, for any nonnegative classical solution $u$ of

$$
-\Delta_{m} u=c(x) u^{p}, \quad x \in B_{1},
$$


u satisfies

$|u(x)|^{(p+1-m) / m}+|\nabla u(x)|^{(p+1-m) /(p+1)} \leq C\left(1+\operatorname{dist}^{-1}\left(x, \partial B_{1}\right)\right), \quad x \in B_{1}$.

Proof. Arguing by contradiction, we suppose that there exist sequences $c_{k}, u_{k}$ verifying (4.1), (4.2) and points $y_{k}$, such that the functions

$$
M_{k}=\left|u_{k}\right|^{(p+1-m) / m}+\left|\nabla u_{k}\right|^{(p+1-m) /(p+1)}
$$

satisfy

$$
M_{k}\left(y_{k}\right)>2 k\left(1+\operatorname{dist}^{-1}\left(y_{k}, \partial B_{1}\right)\right) \geq 2 k \operatorname{dist}^{-1}\left(y_{k}, \partial B_{1}\right) .
$$

By the doubling lemma in [20, Lemma 5.1], there exists $x_{k}$ such that

$$
M_{k}\left(x_{k}\right) \geq M_{k}\left(y_{k}\right), \quad M_{k}\left(x_{k}\right)>2 k \operatorname{dist}^{-1}\left(x_{k}, \partial B_{1}\right),
$$

and

$$
M_{k}(z) \leq 2 M_{k}\left(x_{k}\right), \quad \text { for all } z \text { such that }\left|z-x_{k}\right| \leq k M_{k}^{-1}\left(x_{k}\right) .
$$

We have

$$
\lambda_{k}:=M_{k}^{-1}\left(x_{k}\right) \rightarrow 0, \quad k \rightarrow \infty,
$$

due to $M_{k}\left(x_{k}\right) \geq M_{k}\left(y_{k}\right)>2 k$.

We use the rescaling

$$
v_{k}=\lambda_{k}^{m /(p+1-m)} u_{k}\left(x_{k}+\lambda_{k} y\right), \quad \tilde{c}_{k}(y)=c_{k}\left(x_{k}+\lambda_{k} y\right) .
$$

Then $\left|v_{k}\right|^{(p+1-m) / m}(0)+\left|\nabla v_{k}\right|^{(p+1-m) /(p+1)}(0)=1$,

$$
\left[\left|v_{k}\right|^{(p+1-m) / m}+\left|\nabla v_{k}\right|^{(p+1-m) /(p+1)}\right](y) \leq 2, \quad|y| \leq k,
$$

due to (4.3), and $v_{k}$ is solution in sense of Definition 1.1 to

$$
-\Delta_{m} v_{k}=\tilde{c}_{k}(y) v_{k}^{p}, \quad|y| \leq k .
$$

On the other hand, thanks to (4.1), we have $C_{2} \leq \tilde{c}_{k} \leq C_{1}$ and, for each $R>0$ and $k \geq k_{0}(R)$ large enough,

$$
\left|\tilde{c}_{k}(y)-\tilde{c}_{k}(z)\right| \leq C_{1}\left|\lambda_{k}(y-z)\right|^{\gamma} \leq C_{1}|y-z|^{\gamma}, \quad|y|,|z| \leq R .
$$

Therefore, by Ascoli's theorem, there exists $\tilde{c}$ in $C\left(\mathbb{R}^{N}\right)$, with $\tilde{c} \geq C_{2}$ such that, after extracting a subsequence, $\tilde{c}_{k} \rightarrow \tilde{c}$ in $C_{l o c}\left(\mathbb{R}^{N}\right)$. Moreover, (4.7) and (4.4) imply that $\left|\tilde{c}_{k}(y)-\tilde{c}_{k}(z)\right| \rightarrow 0$ as $k \rightarrow \infty$, so that the function $\tilde{c}$ is actually a constant $C>0$.

By using the regularity results in [29] (see also [8]), we deduce that there exists $\beta \in(0,1)$ such that $v_{k}$ is bounded in $C_{\text {loc }}^{1+\beta}\left(\mathbb{R}^{N}\right)$. Then up to a subsequence, $v_{k}$ converges in $C_{\text {loc }}^{1}\left(\mathbb{R}^{N}\right)$ to a nonnegative solution $v$ (in sense of Definition 1.1), such that

$$
-\Delta_{m} v=C v^{p}, \quad y \in \mathbb{R}^{N} .
$$

Moreover, $|v|^{(p+1-m) / m}(0)+|\nabla v|^{(p+1-m) /(p+1)}(0)=1, v$ is thus nontrivial. This contradicts the Liouville-type theorem (see [25, Theorem II(c)]) and concludes the proof. 
Proof of Proposition 1.5. Assume either $\Omega=\left\{x \in \mathbb{R}^{N} ; 0<|x|<\rho\right\}$ and $0<\left|x_{0}\right|<\rho / 2$, or $\Omega=\left\{x \in \mathbb{R}^{N} ;|x|>\rho\right\}$ and $\left|x_{0}\right|>2 \rho$. We denote

$$
R=\frac{1}{2}\left|x_{0}\right|
$$

and observe that, for all $y \in B_{1}, \frac{\left|x_{0}\right|}{2}<\left|x_{0}+R y\right|<\frac{3\left|x_{0}\right|}{2}$, so that $x_{0}+R y \in \Omega$ in either case. Let us thus define

$$
U(y)=R^{\frac{m+a}{p+1-m}} u\left(x_{0}+R y\right) .
$$

Then $U$ is a solution of

$$
-\Delta_{m} U=c(y) U^{p}, \quad y \in B_{1}, \quad \text { with } c(y)=\left|y+\frac{x_{0}}{R}\right|^{a} .
$$

Notice that $\left|y+\frac{x_{0}}{R}\right| \in[1,3]$ for all $y \in \bar{B}_{1}$. Moreover $\|c\|_{C^{1}\left(\bar{B}_{1}\right)} \leq C(a)$. Then applying Lemma 4.1, we have $U(0)+|\nabla U(0)| \leq C$. Consequently,

$$
u\left(x_{0}\right) \leq C R^{-\frac{m+a}{p+1-m}}, \quad\left|\nabla u\left(x_{0}\right)\right| \leq C R^{-\frac{p+1+a}{p+1-m}},
$$

which yields the desired conclusion.

\section{Proof of Theorem 1.3}

Suppose there exists a positive solution $u$ to $(1.1)$ in $\mathbb{R}^{N}$. We set

$$
F(R)=\int_{B_{R}}|x|^{a} u^{p+1} d x .
$$

By Pohozaev identity, we have

$$
F(R) \leq C\left(G_{1}(R)+G_{2}(R)+G_{3}(R)\right),
$$

where $G_{1}, G_{2}, G_{3}$ are defined in (3.1)-(3.3) Now, by (1.6) in Proposition 1.5, we have

$$
u(x) \leq C|x|^{-\alpha} \quad \text { and } \quad|\nabla u(x)| \leq C|x|^{-1-\alpha}, \quad x \neq 0 .
$$

Due to $p<p_{S}(m, a)$, it follows that

$$
G_{1}(R)+G_{2}(R) \leq C R^{N-m-m \alpha} \rightarrow 0, \quad \text { as } R \rightarrow \infty .
$$

Therefore, $u \equiv 0$ by (4.9): contradiction.

\section{References}

[1] Bidaut-Véron, M.F., García-Huidobro, M.: Regular and singular solutions of a quasilinear equation with weights. Asymptot. Anal. 28(2), 115-150 (2001)

[2] Byeon, J., Wang, Z.-Q.: On the Hénon equation: asymptotic profile of ground states. II. J. Differ. Equ. 216(1), 78-108 (2005)

[3] Byeon, J., Wang, Z.-Q.: On the Hénon equation: asymptotic profile of ground states. I. Ann. Inst. H. Poincaré Anal. Non Linéaire 23(6), 803-828 (2006)

[4] Cao, D., Peng, S., Yan, S.: Asymptotic behaviour of ground state solutions for the Hénon equation. IMA J. Appl. Math. 74(3), 468-480 (2009) 
[5] Carrião, P.C., de Figueiredo, D.G., Miyagaki, O.H.: Quasilinear elliptic equations of the Henon-type: existence of non-radial solutions. Commun. Contemp. Math. 11(5), 783-798 (2009)

[6] Cauchy, A.: Mémoires sur les fonctions complémentaires. C. R. Acad. Sci. Paris 19, 1377-1384 (1844)

[7] Clément, P., de Figueiredo, D.G., Mitidieri, E.: Quasilinear elliptic equations with critical exponents. Topol. Methods Nonlinear Anal. 7(1), 133-170 (1996)

[8] DiBenedetto, E.: $C^{1+\alpha}$ local regularity of weak solutions of degenerate elliptic equations. Nonlinear Anal. 7(8), 827-850 (1983)

[9] Gidas, B., Spruck, J.: Global and local behavior of positive solutions of nonlinear elliptic equations. Commun. Pure Appl. Math. 34(4), 525-598 (1981)

[10] Guedda, M., Véron, L.: Quasilinear elliptic equations involving critical Sobolev exponents. Nonlinear Anal. 13(8), 879-902 (1989)

[11] Hashimoto, T.: Nonexistence of weak solutions of quasilinear elliptic equations with variable coefficients. In: Proceedings of 7th AIMS Conference on Dynamical Systems, Differential Equations and Applications. Discrete Contin. Dyn. Syst. Suppl., pp. 349-358 (2009)

[12] Hénon, M.: Numerical experiments on the stability of spherical stellar systems. Astronom. Astrophys. 24, 229-238 (1973)

[13] Il'yasov, Y.Sh., Takáč, P.: Optimal $W_{\text {loc }}^{2,2}$-regularity, Pohozhaev's identity, and nonexistence of weak solutions to some quasilinear elliptic equations. J. Differ. Equ. 252(3), 2792-2822 (2012)

[14] Kawano, N., Ni, W.-M., Yotsutani, S.: A generalized Pohozaev identity and its applications. J. Math. Soc. Jpn. 42(3), 541-564 (1990)

[15] Liouville, J.: Remarques de M. Liouville sur "Construction géométrique des amplitudes dans les fonctions elliptiques" par M. Charles. C. R. Acad. Sci. Paris 19, 1261-1263 (1844)

[16] Mitidieri, E., Pohozaev, S.I.: A priori estimates and the absence of solutions of nonlinear partial differential equations and inequalities. Tr. Mat. Inst. Steklova 234, 1-384 (2001)

[17] Ni, W.M.: A nonlinear Dirichlet problem on the unit ball and its applications. Indiana Univ. Math. J. 31(6), 801-807 (1982)

[18] Ôtani, M.: Existence and nonexistence of nontrivial solutions of some nonlinear degenerate elliptic equations. J. Funct. Anal. 76(1), 140-159 (1988)

[19] Phan, Q.H., Souplet, P.: Liouville-type theorems and bounds of solutions of Hardy-Hénon equations. J. Differ. Equ. 252(3), 2544-2562 (2012)

[20] Poláčik, P., Quittner, P., Souplet, P.: Singularity and decay estimates in superlinear problems via Liouville-type theorems. I. Elliptic equations and systems. Duke Math. J. 139(3), 555-579 (2007) 
[21] Pucci, P., García-Huidobro, M., Manásevich, R., Serrin, J.: Qualitative properties of ground states for singular elliptic equations with weights. Ann. Mat. Pura Appl. (4) 185(suppl.), S205-S243 (2006)

[22] Pucci, P., Servadei, R.: Existence, non-existence and regularity of radial ground states for $p$-Laplacian equations with singular weights. Ann. Inst. H. Poincaré Anal. Non Linéaire 25(3), 505-537 (2008)

[23] Serra, E.: Non radial positive solutions for the Hénon equation with critical growth. Calc. Var. Partial Differ. Equ. 23(3), 301-326 (2002)

[24] Serrin, J., Zou, H.: Non-existence of positive solutions of Lane-Emden systems. Differ. Integral Equ. 9(4), 635-653 (1996)

[25] Serrin, J., Zou, H.: Cauchy-Liouville and universal boundedness theorems for quasilinear elliptic equations and inequalities. Acta Math. 189(1), 79-142 (2002)

[26] Smets, D., Willem, M.: Partial symmetry and asymptotic behavior for some elliptic variational problems. Calc. Var. Partial Differ. Equ. 18(1), 57-75 (2003)

[27] Smets, D., Willem, M., Su, J.: Non-radial ground states for the Hénon equation. Commun. Contemp. Math. 4(3), 467-480 (2002)

[28] Souplet, P.: The proof of the Lane-Emden conjecture in four space dimensions. Adv. Math. 221(5), 1409-1427 (2009)

[29] Tolksdorf, P.: Regularity for a more general class of quasilinear elliptic equations. J. Differ. Equ. 51(1), 126-150 (1984)

Quoc Hung Phan

Institute of Research and Development

Duy Tan University

Da Nang

Vietnam

e-mail: phanquochung@dtu.edu.vn

Anh Tuan Duong

Department of Mathematics

Hanoi National University of Education

136 Xuan Thuy Street

Cau Giay District

Hanoi

Vietnam

e-mail: tuanda@hnue.edu.vn

Received: 11 March 2015.

Accepted: 27 July 2015. 